\title{
A retrospective study of 109 dogs with mandibular fractures
}

\author{
A. M. Kitshoff ${ }^{1,2} ;$ H. de Rooster'; S. M. Ferreira ${ }^{3} ;$ G. Steenkamp ${ }^{4}$ \\ 'Section Small Animal Surgery, Department of Companion Animal Clinical Studies, Faculty of Veterinary Science, University of Pretoria, Onderstepoort, South Africa; \\ ${ }^{2}$ Department of Small Animal Medicine and Clinical Biology, Faculty of Veterinary Medicine, Ghent University, Merelbeke, Belgium; \\ ${ }^{3}$ Savanna Research Unit, Scientific Services, Conservation Services Division, South African National Parks, Skukuza, South Africa; \\ ${ }^{4}$ Maxillofacial Surgery and Dentistry Clinic, Sec-tion Small Animal Surgery, Department Companion Animal Clinical Studies, Faculty of Veterinary Science, University of Pretoria, \\ Onderstepoort, South Africa \\ Correspondence to: \\ Adriaan M. Kitshoff, BVSc (Hons) Department of Small Animal Medicine and Clinical Biology Faculty of Veterinary Medicine, Ghent University, Salisburylaan 133 \\ Merelbeke, B-9820. Belgium. Phone: +329264 7700 Fax: +32 92647791 E-mail: adriaan.kitshoff@ugent.be
}

\begin{abstract}
Summary
Objective: To determine patient factors and fracture morphology of dogs presented with mandibular fractures to a small animal referral centre in South Africa.

Methods: Patient data on age, sex, breed and aetiology of dogs with mandibular fractures were recorded. The fractures were clas-sified according to the anatomical location, displacement, fracture type, fracture line direction, periodontal pathology, and whether there were teeth in the fracture line or not by evaluation of preoperative radiographs. Clinical observations indicated whether these fractures were open or closed. Results: In total, 109 dogs with 135 mandibular fractures were included in the study.

Small breed dogs and dogs less than eight months of age predominated (102/109). Dog fights were the most common aetiology in this study (68/109). The molar region was the most commonly affected region (56/135). Evaluation of the radiographs revealed that transverse (73/135), relatively unstable (116/135), and displaced (112/135) fractures were the most common. The majority of fractures involved teeth in the fracture line (100/135), with the first molar frequently involved (54/135). The majority of fractures were open (104/135).

Clinical significance: The results obtained from this study may be used to guide patient and fracture morphology selection in bio-mechanical studies of mandibular fracture repair techniouses Screening of this natient nonulation mav insnire the search for new treatment options for mandibular fracture repair in South Africa.
\end{abstract}

\section{Keywords}

Mandibular, fracture, classification, molar, open fracture, small breed.

\section{Introduction}

The bones' ability to absorb forces will de-pend on the inherent characteristics of the bone such as mineral content, and presence of pathology, as well as the species type, age, and sex of the animal $(1,2)$. Inorganic structural materials provide compressive strength and are responsible for the stiff-ness whereas organic components deter-mine the tensile properties (1). If bone can-not resist the forces applied to it, it will fracture $(1,3)$. Fractures can either be a complete or incomplete break in the con-tinuity of the bone $(1,3)$.
Fracture morphology depends on the aetiology and patient factors. In humans, patient factors depend mainly on anatomical characteristics like the relative strength of certain regions of the bone, size and shape of the bone, and muscle attachments (4). Fracture morphology also depends on the characteristics of the force such as direction, rate, amount and nature, as well as the spatial relationship of the bone to the force (4).

Mandibular fractures are reported to have a high incidence in male dogs less than twelve months of age $(5,6)$. The mandibular body is most commonly involved in mandibular fractures $(5,6)$.

Anatomical location and conformation of these fractures can play an important role in the selection of treatment methods and can aid in prognostication of fractures $(7,8)$.

Although previous retrospective studies of oral fractures in dogs exist, none of these describe the fracture morphology in detail $(5,6)$. In the present study, dogs with mandibular fractures presented to a South African referral clinic were studied.

\section{Materials and methods}

The case records and radiographs of dogs with mandibular fractures that were presented to the Dentistry and Maxillofacial Surgery Clinic at the Onderstepoort Veterinary Academic Hospital in South Africa between January 2001 and December 2009 were reviewed retrospectively. 


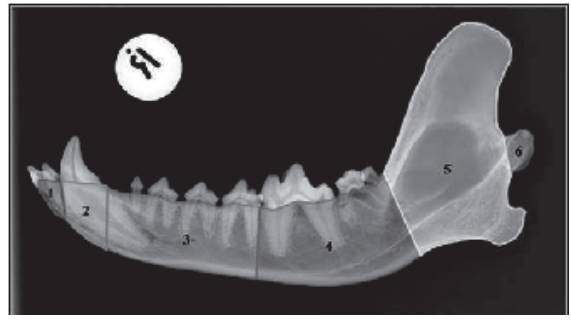

Figure 1 Anatomical regions used to classify the mandibular fracture locations: 1) Incisive region; 2) Canine region; 3) Premolar region;

4) Molar region; 5) Ramus region; and 6) Condylar process.

Data recorded from the patient files included sex (entire or neutered), age (months), weight, and breed. The information recorded in the patient files was also used to determine whether the fractures were open or closed. Aetiological factors were classified as dog fights, road traffic accidents, idiopathic, pathologic or other. All preoperative radiographs were evaluated by the first author (AK). The data recorded from the radiographic re-evaluation included anatomical location ( Figure 1), fracture conformation, unilateral or bilateral involvement, the presence of tooth roots in the fracture line, displacement, fracture stability, and radiographic evidence of pathology ( Appendix Table 1 available online at www.vcot-online.com). Patients with incomplete patient records were excluded from the study.

Categorical data were evaluated using Pearson's Chi-squared test and continuous data using the paired $t$-test. The age groups in this study were compared to studies done by Umphlet et al. and Lopes et al. by calculating the coefficient of determination $\left(\mathrm{R}^{2}\right)(5,6)$. The breeds of the dogs that were presented to the hospital for mandibular fractures were compared to the entire patient population for the same time period, and test statistics were performed to evaluate whether certain breeds were presented more than expected. All calculations were done using a commercial spreadsheet program ${ }^{\mathrm{a}}$. Statistical significance was set at $\mathrm{p} \leq 0.05$.

\section{Results}

The case records of 119 dogs that were presented between January 2001 and December 2009 were identified and reviewed. Due to incomplete records 10 patients were excluded. A total of 135 mandibular fractures were present in the 109 dogs included in this study.

Fifty-seven percent of the dogs were under the age of twelve months at the time of fracture management ( $\triangleright$ Figure 2). In this age group, males $(\mathrm{n}=32)$ and females $(n=29)$ were almost equally represented (Chi-squared test: $\chi^{2}=0.067 ; p=0.80$ ). In the patients older than twelve months, a male: female ratio of 1.9:1 was observed although the difference was not statistically significant (Chi-squared test: $\chi^{2}=3.000$; $p$ $=0.08)$. In this group of patients, $69 \%$ of the males and $6.25 \%$ of the females were entire. The age distribution in the current study compared more closely to the findings of the study done by Umphlet et al. ( $\mathrm{R}^{2}$

Excel 2010: Microsoft, Redmond, Washington, United States
$=0.92)$ than to the one done by Lopes et al. $\left(\mathrm{R}^{2}=0.34\right)(5,6)$.

A high incidence of mandibular fractures was noted in small breed dogs (77/109; $70.6 \%$ ). The mean body weight of patients in this study was $6.46 \mathrm{~kg}$ (standard deviation \pm $5.4 \mathrm{~kg}$ ) and the median was $5.2 \mathrm{~kg}$. Of the total dog population that was presented to the hospital during the same time period, 1023/2321 (44.1\%) were small breeds. The five breeds that had the highest incidence of mandibular fractures were: Yorkshire Terriers (16\%), Dachshunds (14\%), Jack Russell Terriers (11\%), Maltese (10\%) and Pekingese $(6 \%)$. These breeds made up $15 \%, 24 \%, 11 \%$, $21 \%$ and $2 \%$ respectively of the total patient population. Test statistics revealed that only Pekingese dogs were presented more often than expected ( $p=0.001)$.

Large breed dogs older than eight months of age only made up six percent of the study population.

Dog fights were the most common aetiology (68/109) of mandibular fractures in this study ( $\triangleright$ Figure 3 ).

The patients with aetiologies that did not fit into any of the groups were classified as 'other'. In this group, mandibular fractures due to gunshot $(\mathrm{n}=3)$, pig bite $(\mathrm{n}=1)$, kick from a horse $(\mathrm{n}=1)$, hit with a metal pole ( $\mathrm{n}$ $=1)$, run over by a wheelbarrow $(n=1)$, and chewing on a hard object in the absence of pathology $(n=1)$, were included. Only one mandibular fracture was caused iatrogenically during extraction of teeth. This fracture was subsequently classified as pathologic due to the radiographic presence of alveolar bone lysis. Pathologic fractures also occurred in three other dogs.

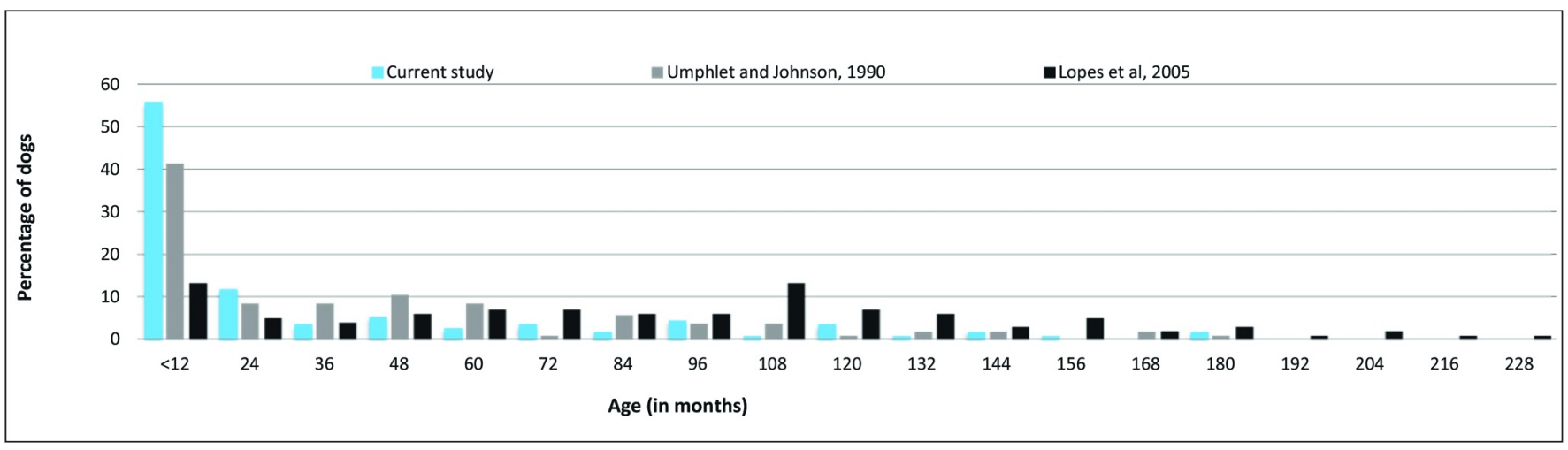

Figure 2 The age distribution of the 109 patients presented to the Dentistry and Maxillofacial Surgery Clinic. Age distributions extracted from Umphlet et al. and Lopes et al. are provided $(5,6)$. 
The incidence of mandibular fractures was highest in the molar region ( Figure 4). Anatomical distribution of mandibular fractures were similar to that noted in two other studies $(5,6)$.

Of the 135 fractures, 104 were open ( Figure 5). There was a high incidence of open fractures in the dentulous portion of the mandible (87/104).

The fracture type was transverse in 73 , short oblique in 48 , oblique in one, comminuted in 11, and incomplete in two of the cases. The fractures were relatively unstable in 116/135 and displaced in 112/135. Almost three quarters of the fractures extended through the alveolus of one or more teeth. A total of 124 teeth were involved in the fracture line with the first molar predominating ( $\triangleright$ Figure 6 ).

In small breed dogs, fractures of the molar, premolar and symphyseal regions predominated compared to large breed dogs where fractures of the ramus, incisive, canine, and condylar process regions were more common (Chi-squared test: $\chi^{2}=$ 23.73; $\mathrm{p}=0.02)$.

Contingency tables also showed that aetiology was associated with fracture type (Chi-squared test: $\chi^{2}=30.27 ; \mathrm{p}=0.02$ ) and anatomical location (Chi squared test: $\chi^{2}=$ 36.36; $\mathrm{p}=0.05$ ). Higher than expected incidences of short oblique fractures in the canine and premolar regions, incomplete fractures of the ramus region, and transverse fractures of the condylar process were noted when the fractures occurred as a result of road traffic accident.

Multiple mandibular fractures were present in 24 of the patients. The multiple fractures included fractures involving both mandibles $(n=14)$, multiple fractures with non-communicating fracture lines of one mandible $(\mathrm{n}=3)$, and mandibular fracture together with symphyseal separation $(n=7)$. Two-thirds of these patients were presented after a dog fight.

\section{Discussion}

Previous studies found that mandibular fractures accounted for $1.6 \%$ to six percent of all fractures in dogs that were presented to veterinary hospitals $(11,12)$. In other studies. the main aetiologies for mandibu-

\section{Figure 3}

Fracture aetiology of the 109 dogs presented to the Dentistry and Maxillofacial Surgery Clinic. RTA = Road traffic accident.

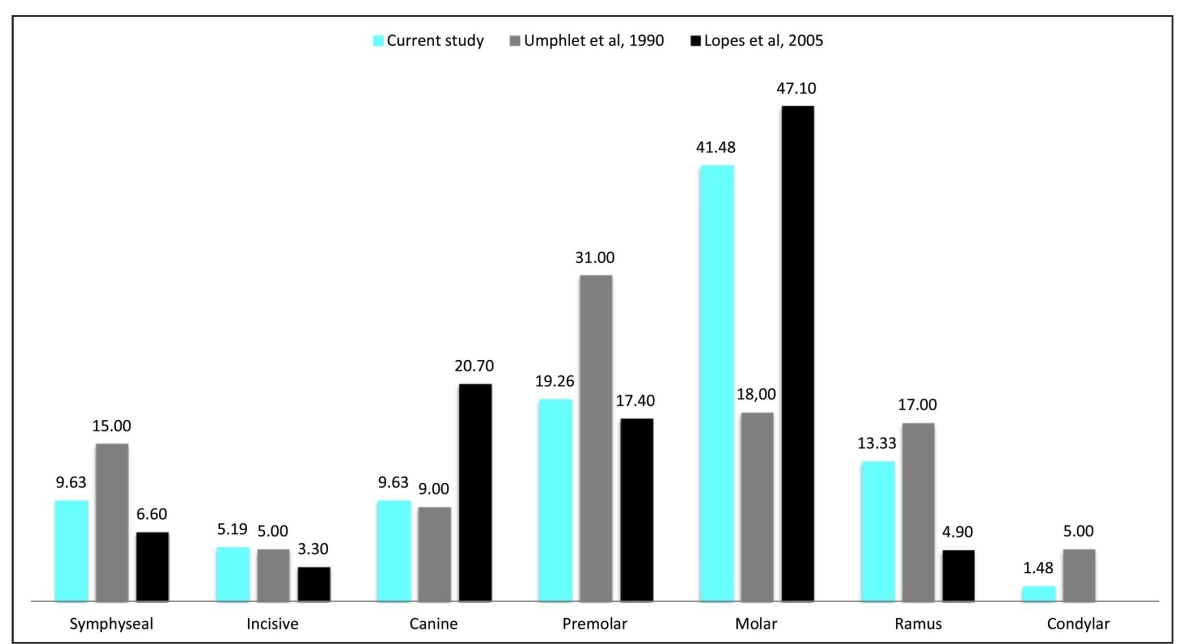

Figure $4 \mathrm{~A}$ comparative presentation of fracture frequencies in the various anatomical locations of the mandible. The comparison takes into consideration this current study as well as those done by Umphlet et al. and Lopes et al. $(5,6)$.

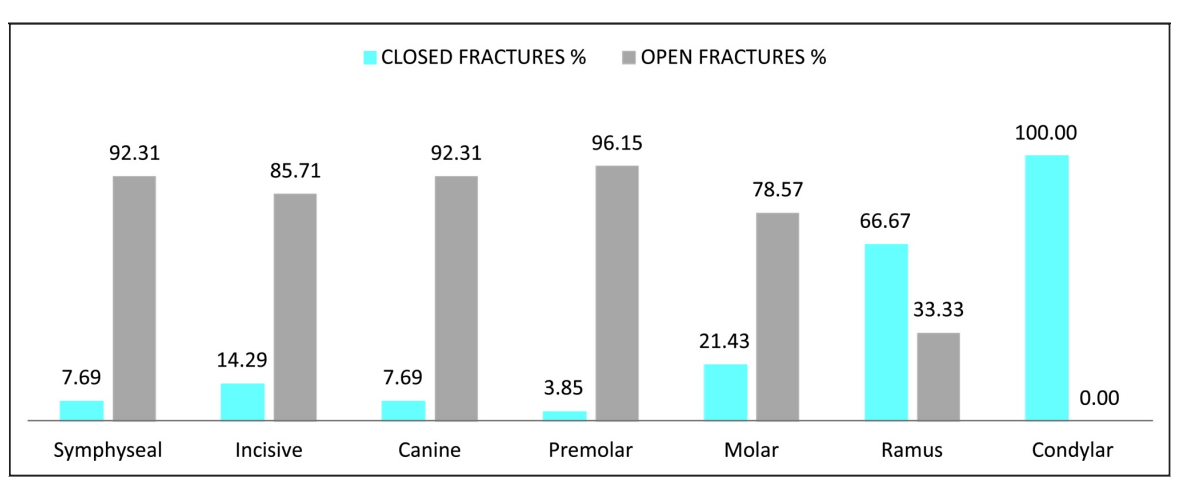

Figure 5 The incidence of open or closed fractures based on the anatomical locations involved.

lar fractures were road traffic accidents, falls from a height, projectile injuries, unknown trauma, and dog fights $(5,6,11)$. In our study, dog fights were more frequently the cause of mandibular fractures $(62 \%)$ than has been previously reported $(19 \%$ $43 \%)(5.6)$.
During dog fights, the head and neck are frequently targeted areas (13). The relative sizes of the victim and the attacker play a significant role in the severity of bite wounds (13). As the size of the animal increases so does its biting forces (14). This makes smaller dogs more prone to fractur- 


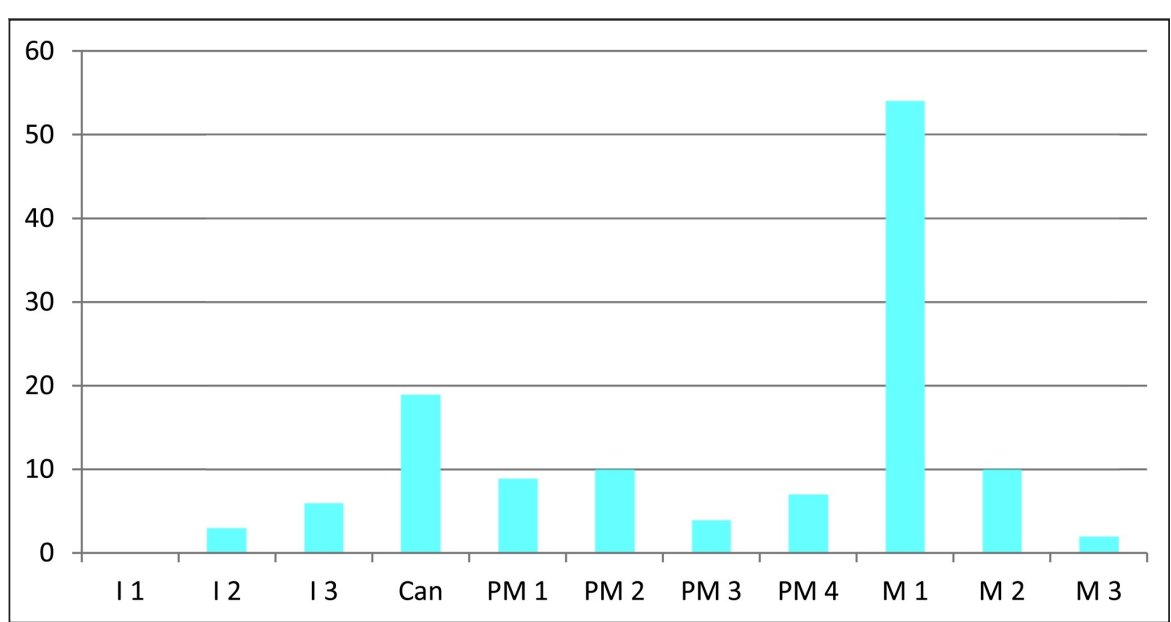

Figure 6 Regional distribution of the 124 teeth in the fracture line of 109 dogs with 135 mandibular fractures. I = incisor, Can = canine, $\mathrm{PM}=$ premolar, $\mathrm{M}=$ molar.

ing their mandibles if bitten by larger dogs compared to when larger dogs are bitten by smaller dogs. The popularity of large breed dogs like Boerboels and multi-dog households (especially mixed large and small breed) in the Onderstepoort area are factors that might explain the higher incidence of mandibular fractures caused by dog bites in this study. It is the authors' opinion that owners in the Onderstepoort region prefer to keep larger guard dogs on their properties together with smaller pets.

Small breed dogs less than 12 months of age were the most common size and age group affected. As dogs mature the bone mineral content, and thus its density, increases (15). Therefore, young dogs may be over-represented as they have relatively low bone density predisposing the bone to failure (16).

Pekingese dogs were presented more than was expected. It is uncertain whether their particular anatomy, behaviour or personality predisposes them to mandibular fractures and further studies would be needed to clarify this.

Of the patients older than 12 months, entire male dogs were over-represented. When compared to castrated males, entire male dogs are involved in more incidents of aggression and roam more frequently (17, 18). Roaming will increase the opportunity for contact with other dogs and with motor vehicles.

On evaluation of preoperative radiographs, only a few patients had lysis of the volved in nearly all the cases. Fractures through the alveolus of the first mandibu- height ratio can act as stress risers, potentially explaining the high incidence of molar region fractures in this study. Of all the fractures that occur in the molar re- lar molar might also be related to early periodontitis that results in weakening of the alveolus and periodontal ligament. This weakening might be the result of periodontal ligament destruction which is a lesion that would not be detectable radiographically.

The nature of the force may have had an influence on the type and location of the fractures in our study.

The low incidence of mandibular fractures through the condylar process was a finding that was similar to another study (5). Research has shown differences in the indentation modulus of the condyles in young and adult dogs (22). It is unknown whether this difference, due to aging, is clinically relevant. During mastication, the shearing action of the carnassial teeth in dogs requires a mild amount of transverse movement of the condyles (23). This minimal movement in the joint and the protective effect of the masticatory muscles might be enough to absorb the forces and thus decrease the incidence of fractures of the condyles. Due to the low number of condylar fractures no significant conclusion could be drawn about the fracture types seen in this region.

The presence of teeth influences the fracture line direction as the fracture line will follow the route of least resistance, resulting in fractures along the tooth roots (24). Likewise the synchondrosis intermandibularis is the weakest area in the symphyseal region as it is composed of fibrocartilage (25). A high incidence of transverse fractures through the symphyseal region and short oblique fractures in the canine and premolar regions support this statement.

The protective and supporting effect of the caudal masticatory muscles probably resulted in the high incidence of incomplete and non-displaced fractures in the ramus region.

Mandibular fractures in the dentulous portion of the mandible were mostly open. Only a thin layer of gingiva covers these regions of the mandible orally. Displacement of the fracture fragments and the fragments sharp edges will easily disrupt the gingiva. 


\section{Conclusion}

Adult large breed dogs were under-represented. The relatively big root of the first molar compared to the mandibular height in small breed dogs might explain the high incidence of molar region fractures.

\section{Conflict of interest}

None declared

\section{References}

1. Doblaré M, García JM, Gómez MJ. Modelling bone tissue fracture and healing: a review. Eng Frac Mech 2004; 71: 1809-1840.

2. Vashishth D. Small animal bone biomechanics. Bone 2008; 43: 794-797.

3. Piermattei DL, Flo GL, DeCamp CE. Fractures and luxations of the mandible and maxilla. In: Brinker, Piermattei, and Flo's Handbook of Small Animal Orthopedics and Fracture Repair. 4th ed. St. Louis, Mo.: Elsevier Saunders; 2006. pg. 717-736.

4. Copcu E, Sisman N, Oztan Y. Trauma and fracture of the mandible. Eur J of Trauma 2004; 30: 110-115.

5. Umphlet RC, Johnson AL. Mandibular fractures in the dog. A retrospective study of 157 cases. J Vet Surg 1990; 19: 272-275.

6. Lopes FM, Gioso MA, Ferro DG, et al. Oral fractures in dogs of Brazil--a retrospective study. J Vet Dent 2005; 22: 86-90.
7. Glyde M, Lidbetter D. Management of fractures of the mandible in small animals. In Practice 2003; 570-585.

8. Vertstraete FJM. Maxillofacial fractures. In: Holmstrom SE, Frost P, Eisner ER, editors.Veterinary Dental Techniques for the Small Animal Practitioner. 3rd ed. Philadelphia: W.B. Saunders; 2004 pg. 559-600.

9. McAllister H, Tobin E. Long bones- fractures. In: Barr F, Kirberger R, editors. BSAVA Manual of Canine and Feline Musculoskeletal Imaging. Cheltenham: British Small Animal Veterinary Association; 2006. pg. 49-70.

10. Buitrago-Téllez CH, Audigé L, Strong B, et al. A comprehensive classification of mandibular fractures: a preliminary agreement validation study. Int J Oral Maxillofac Surg 2008; 37: 1080-1088.

11. Wong WT. A survey of fractures in the dog and cat in Malaysia. Vet Rec 1984; 115: 273-274.

12. Phillips IR. A survey of bone fractures in the dog and cat. J Small Anim Pract 1979; 20: 661-674.

13. Shamir MH, Leisner S, Klement E, et al. Dog bite wounds in dogs and cats: a retrospective study of 196 cases. J of Vet Med 2002; 49: 107-112.

14. Ellis JL, Thomason J, Kebreab E, et al. Cranial dimensions and forces of biting in the domestic dog. J Anat 2009; 214: 362-373.

15. Lorinson K, Loebcke S, Skalicky M, et al. Signalment differences in bone mineral content and bone mineral density in canine appendicular bones. A cadaveric study. Vet Comp Orthop Traumatol 2008; 21: 147-151.

16. Pietrzak WS, Sarver DR, Kohn DH. Fatigue testing of bioabsorbable screws in a synthetic bone substrate. In: An YH, Draughn RA, editors. Mechanical Testing of Bone and the Bone-Implant Interface. Washington: CRC press; 1999. pg. 581-91.
17. Hopkins SG, Schubert TA, Hart BL. Castration of adult male dogs: effects on roaming, aggression, urine marking, and mounting. J Am Vet Med Assoc 1976; 168: 1108-1110.

18. Overall KL, Love M. Dog bites to humans-demography, epidemiology, injury, and risk. J Am Vet Med Assoc 2001; 218: 1923-1934.

19. Erikson T. Physical orodental conditions. In: Tutt C, Deeprose J, Crossley DA, editors. BSAVA Manual of Canine and Feline Dentistry. $3^{\text {rd }}$ ed. Cheltenham: British Small Animal Veterinary Association; 2007.pg. 148-159.

20. Smith MM, Massoudi LM. Potential attachment area of the first mandibular molar in dogs. Am J Vet Res 1992; 53: 258-261.

21. Gioso MA, Shofer F, Barros PS, et al. Mandible and mandibular first molar tooth measurements in dogs: relationship of radiographic height to body weight. J Vet Dent 2001; 18: 65-68.

22. Huja SS, Rummel AM, Beck FM. Changes in mechanical properties of bone within the mandibular condyle with age. J of Morph 2008; 269: 138-143.

23. Vollmerhaus B, Roos $\mathrm{H}$. The transverse movement of the temporo-mandibular joint (translation movement) of the dog, also with reference to dysplasia of this joint in the dachshund. Anat Histol Embryol 1996; 25: 145-149.

24. Scott HW. The skull and mandible. In: Coughlan AR, Miller A, editors. BSAVA Manual of Small Animal Fracture Repair and Management. Cheltenham: British Small Animal Veterinary Association; 1998. pg. 115-129.

25. Verstraete FJM. Maxillofacial fractures. In: Slatter DH, editor. Textbook of Small Animal Surgery. 3rd ed. Philadelphia, PA: Saunders; 2003. pg. 2190-2207. 